\title{
Coenzyme Q Biosynthesis: An Update on the Origins of the Benzenoid Ring and Discovery of New Ring Precursors
}

\author{
Lucía Fernández-del-Río (D) and Catherine F. Clarke *(D) \\ Department of Chemistry and Biochemistry and the Molecular Biology Institute, University of California, \\ Los Angeles, CA 90095-1569, USA; luciafernandezdelrio@gmail.com \\ * Correspondence: cathy@chem.ucla.edu
}

check for updates

Citation: Fernández-del-Río, L.; Clarke, C.F. Coenzyme Q

Biosynthesis: An Update on the Origins of the Benzenoid Ring and Discovery of New Ring Precursors. Metabolites 2021, 11, 385. https:// doi.org/10.3390/metabo11060385

Academic Editors: Ajit Divakaruni, Martina Wallace and

Amedeo Lonardo

Received: 15 May 2021

Accepted: 8 June 2021

Published: 14 June 2021

Publisher's Note: MDPI stays neutral with regard to jurisdictional claims in published maps and institutional affiliations.

Copyright: (c) 2021 by the authors. Licensee MDPI, Basel, Switzerland. This article is an open access article distributed under the terms and conditions of the Creative Commons Attribution (CC BY) license (https:/ / creativecommons.org/licenses/by/ $4.0 /)$.

\begin{abstract}
Coenzyme Q (ubiquinone or CoQ) is a conserved polyprenylated lipid essential for mitochondrial respiration. CoQ is composed of a redox-active benzoquinone ring and a long polyisoprenyl tail that serves as a membrane anchor. A classic pathway leading to CoQ biosynthesis employs 4-hydroxybenzoic acid (4HB). Recent studies with stable isotopes in E. coli, yeast, and plant and animal cells have identified CoQ intermediates and new metabolic pathways that produce $4 \mathrm{HB}$. Stable isotope labeling has identified para-aminobenzoic acid as an alternate ring precursor of yeast CoQ biosynthesis, as well as other natural products, such as kaempferol, that provide ring precursors for CoQ biosynthesis in plants and mammals. In this review, we highlight how stable isotopes can be used to delineate the biosynthetic pathways leading to CoQ.
\end{abstract}

Keywords: coenzyme Q; ubiquinone; stable isotopes; biosynthesis; 4-hydroxybenzoic acid; $p$ aminobenzoic acid; natural products; polyphenols; kaempferol

\section{Introduction}

Coenzyme Q (CoQ or ubiquinone) is an essential redox-active lipid that functions in cellular energy metabolism in eukaryotes and numerous bacterial species [1-3]. The redox chemistry of $\mathrm{CoQ}$ and $\mathrm{CoQH}_{2}$ (ubiquinol, a hydroquinone) allows it to serve as a vital electron carrier in the respiratory chain. CoQ accepts electrons and protons from Complex I or Complex II and donates them to Complex III, thereby establishing a proton gradient across the mitochondrial inner membrane that ultimately leads to the production of ATP [4]. CoQ also functions as an essential electron and proton acceptor for other dehydrogenases required for fatty acid metabolism, pyrimidine biosynthesis, and the oxidation of sulfide, proline, and glycerol-3-phosphate [2-5]. Besides these metabolic roles, $\mathrm{CoQH}_{2}$ doubles as a crucial antioxidant that protects lipids, proteins, and DNA from oxidative damage [4].

The overall architecture of CoQ biosynthesis is similar in prokaryotes and eukaryotes: a long polyisoprenoid lipid tail is coupled to a benzenoid precursor, and the benzenoid ring is further modified through successive steps to yield the final product [2,6] (Figure 1A). The universal aromatic ring precursor of CoQ is 4-hydroxybenzoic acid (4HB) [3]; the origins of $4 \mathrm{HB}$ and alternative ring precursors will be discussed throughout this review. The isoprene carbon units for making the CoQ side-chain are derived from the mevalonate pathway in eukaryotes and some prokaryotes [7,8], or the deoxyxylulose-5-phosphate pathway in prokaryotes, some protozoa, and plants [9-11] (Figure 1A,B). CoQ is anchored at the midplane of the phospholipid bilayer by a long polyisoprenoid tail. The number of isoprene units ( $\mathrm{n}$ ) that comprise the polyisoprenoid tail of $\mathrm{CoQ}_{\mathrm{n}}$ is determined by a species-specific polyprenyl diphosphate synthase (IspB in Escherichia coli (E. coli), Coq1 in Saccharomyces cerevisiae (S. cerevisiae), PDSS1-PDSS2 in mammals, and AtSPS3 in Arabidopsis thaliana (Arabidopsis)) [3,12-14] (Step A, Figure 1A,B). Therefore, $\mathrm{CoQ}_{8}$ is the major CoQ isoform in E. coli, $\mathrm{CoQ}_{6}$ in $S$. cerevisiae, CoQ 9 in rodents and plants, and $\mathrm{CoQ}_{10}$ in humans and Schizosaccharomyces pombe (S. pombe). This step and the rest of CoQ biosynthesis is reported to occur in the mitochondria, with the exception of E. coli, where $\operatorname{CoQ}_{8}$ has been recently 
proposed to be synthesized in the cytosol on a soluble metabolon and then trafficked to the plasma membrane $[15,16]$. Then, UbiA/Coq2/COQ2/AtPPT1 (E. coli, S. cerevisiae, mammals, and Arabidopsis, respectively) catalyze the attachment of the polyisoprenoid tail to position C-3 of the ring precursor [3,12-14] (Step B, Figure 1A,B). In S. cerevisiae and S. pombe, Coq2 also prenylates the ring of para-aminobenzoic acid ( $p \mathrm{ABA})$, which serves as an alternative $\mathrm{CoQ}$ ring precursor [17-19] (Figure 1A,B).

A

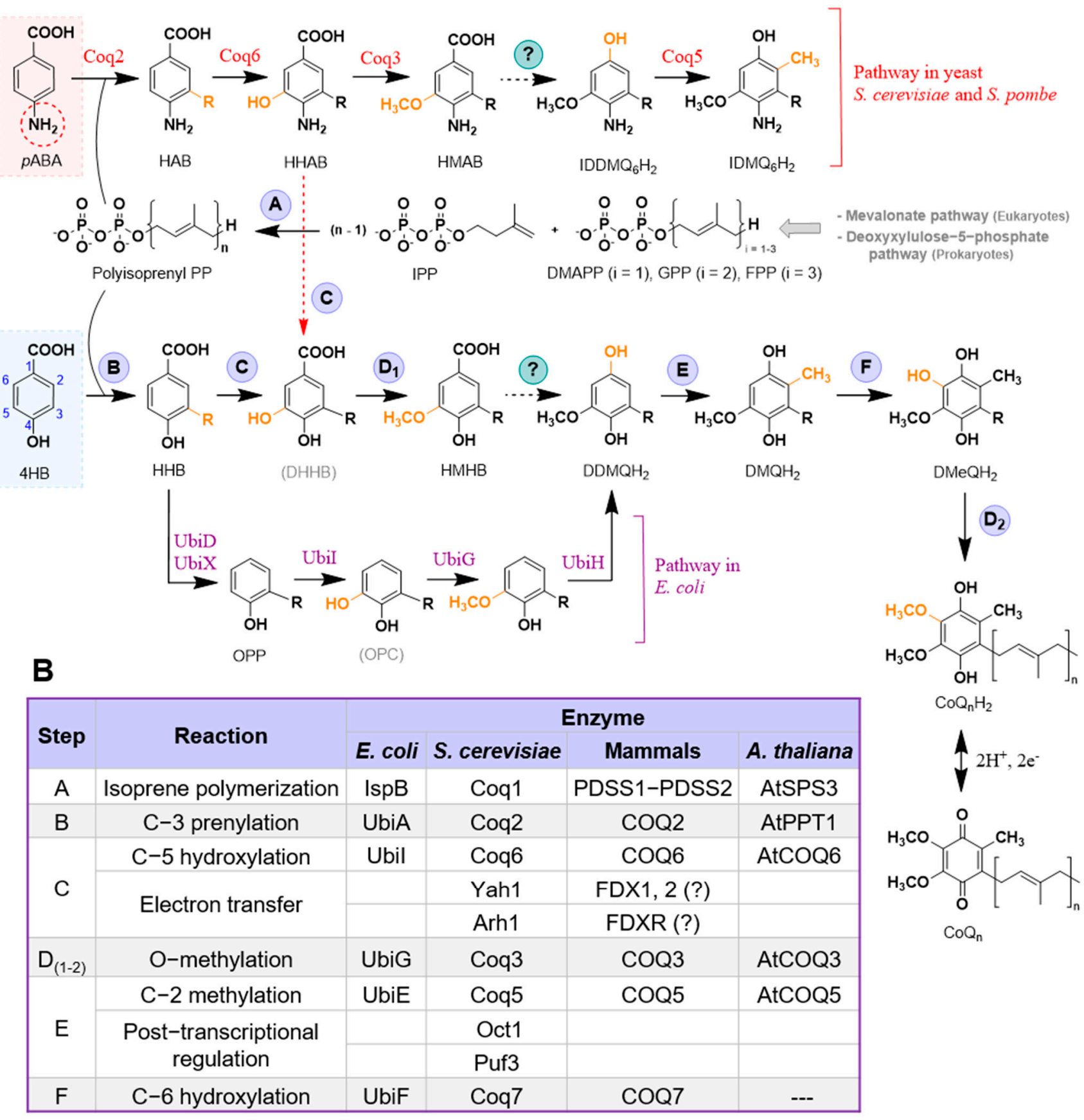

Figure 1. Current model of CoQ biosynthesis. (A) The primary CoQ pathway that is conserved among different organisms is depicted, starting from the synthesis of the polyisoprenoid tail and its attachment to the universal precursor $4 \mathrm{HB}$. Each step has been assigned a specific letter that refers to the table in (B). Steps labeled with a (?) indicate that the responsible enzyme(s) is unknown. Specific steps in E. coli CoQ biosynthesis that differ from the standard pathway are shown. The utilization of $p$ ABA 
in S. cerevisiae and S. pombe as an alternative precursor and the intermediates generated from its utilization are also depicted. (B) This table summarizes each reaction and its corresponding enzyme in different organisms. Abbreviations: 4HB, 4-hydroxybenzoic acid; $p \mathrm{ABA}$, para-aminobenzoic acid; $\mathrm{DDMQH}_{2}$, 3-hexaprenyl-5-methoxy-1,4-benzenediol; DHHB, 3-hexaprenyl-4,5-dihydroxybenzoic acid; DMAPP, dimethylallyl pyrophosphate; $\mathrm{DMeQH}_{2}$, 3-hexaprenyl-2-methyl-5methoxy-1,4,6-benzenetriol; $\mathrm{DMQH}_{2}$, 3-hexaprenyl-2-methyl-5-methoxy-1,4-benzenediol; GPP, geranyl pyrophosphate; HAB, 4-amino-3-hexaprenylbenzoic acid; HHAB, 4-amino-3-hexaprenyl-5-hydroxybenzoic acid; HHB, 3-hexaprenyl4HB; HMAB, 4-amino-3-hexaprenyl-5-methoxybenzoic acid; HMHB, 3-hexaprenyl-4-hydroxy-5-methoxybenzoic acid; IDDMQ ${ }_{6} \mathrm{H}_{2}$, 4-amino-3-hexaprenyl-5-methoxyphenol; IDMQ ${ }_{6} \mathrm{H}_{2}$, 4-amino-3-hexaprenyl-2-methyl-5-methoxyphenol; IPP, isopentenyl pyrophosphate; OPC, 3-octaprenyl catechol; OPP, 3-octaprenylphenol. Unless otherwise noted, the acronyms refer to hexaprenyl S. cerevisiae intermediates; in S. pombe $\mathrm{n}=10$. Intermediates denoted with acronyms in brackets represent those that have not yet been experimentally detected.

Starting from the first prenylated intermediate (3-hexaprenyl-4HB; HHB) the ring is further modified by successive reactions. In eukaryotes, $C-5$ hydroxylation is catalyzed by Coq6/COQ6/AtCOQ6 [2,3,7,13,14,20] (Step C, Figure 1A,B). Coq6 is also necessary for the $\mathrm{C}-4$ deamination in $\mathrm{S}$. cerevisiae when $p \mathrm{ABA}$ is used as the aromatic ring precursor $[3,20]$. Two additional proteins were described to be necessary for the correct functionality of Coq6: Yah1, ferredoxin, and Arh1, ferredoxin reductase [21]. However, whether the mammalian homologs-FDX1, 2 and FDXR-perform the same function is still unknown [2,3]. In prokaryotes, UbiI catalyzes the same C-5 hydroxylation reaction, but it is preceded by a C-1 decarboxylation catalyzed by UbiD and UbiX [1,12] (Figure 1A,B). Then, UbiG/Coq3/COQ3/AtCOQ3 catalyze the C-5 O-methylation $[2,3,7,13,14]$ (Step $\mathrm{D}_{1}$, Figure 1A,B). In eukaryotes, the enzyme(s) that catalyze(s) the following decarboxylation and hydroxylation reactions have not been identified yet. In prokaryotes, $\mathrm{UbiH}$ performs this specific hydroxylation $[1,12]$ (Figure 1A,B). UbiE/Coq5/COQ5/AtCOQ5 catalyze the subsequent C-2 methylation $[2,3,7,13,14]$ (Step E, Figure 1A,B). In S. cerevisiae, the posttranscriptional modifications of Coq5 performed by Oct1 and Puf3 have been described to be necessary for the correct functioning of the protein [22-24]. UbiF/Coq7/COQ7 catalyze the C-6 hydroxylation (Step F, Figure 1A,B) and UbiG/Coq3/COQ3/AtCOQ3 catalyze the final C-6 O-methylation to yield CoQ (Step $\mathrm{D}_{2}$, Figure 1A,B). No homolog of COQ7 has been identified in plants [13].

Interestingly, a recent study suggested an alternative order for some steps of the $\mathrm{CoQ}$ biosynthetic pathway. Due to the accumulation of 3-decaprenyl-1,4-benzoquinol (4- $\left.\mathrm{HP}_{10}\right)$ in human cells lacking COQ6, the decarboxylation and hydroxylation reactions are proposed to occur before the action of COQ6 (Figure 2A) [25]. Another possibility is that the C1 decarboxylation and hydroxylation occur independently of the hydroxylation catalyzed by COQ6 [25]. It is noteworthy that the analogous metabolite, 3-hexaprenyl-1,4-benzoquinol $\left(4-\mathrm{HP}_{6}\right)$ (Figure $\left.2 \mathrm{~A}\right)$, was originally detected in yeast mutants that were deficient in Yah1 or Arh1 [18], and was also detected in certain S. cerevisiae coq6 point mutants [21], and in coq6 and coq9 null mutants over-expressing Coq8 [26]. In the yeast studies, $4-\mathrm{HP}_{6}$ was presumed to be a dead-end metabolite. Further work will be required to determine whether or not $4-\mathrm{HP}_{6}$ and / or $4-\mathrm{HP}_{10}$ are productive intermediates. It is sometimes the case that metabolites accumulate because they are not productive (e.g., IDMQ in yeast and the amino-containing octaprenyl metabolites in E. coli) [27]. Conversely, other metabolites have not yet been detected (e.g., octaprenyl catechol in E. coli [28]), or have not been detected reproducibly (e.g., DHHB in S. cerevisiae [29-33]). These intermediates may elude detection due to rapid metabolism and/or instability. 
A

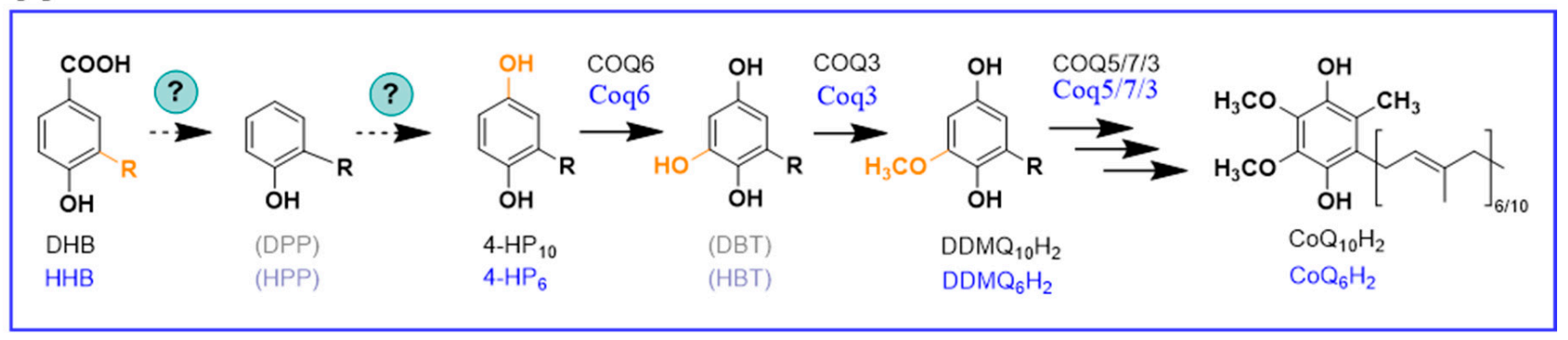

B

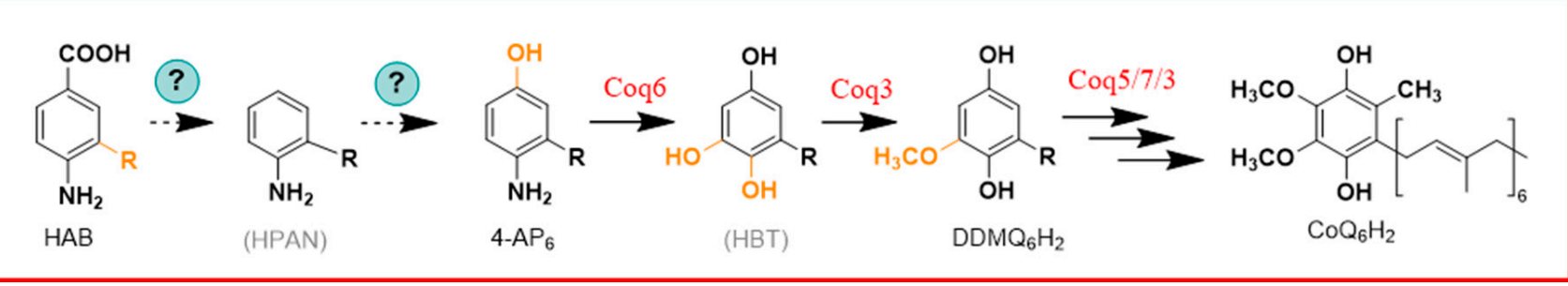

Figure 2. Alternative order of some reactions in the CoQ biosynthesis pathway. (A) Diagram representing the alternative order in some steps in mammalian CoQ biosynthesis (black labels) as proposed in [25], as well as the hypothetical steps in yeast CoQ biosynthesis (blue labels) based on the detection of 4- $\mathrm{HP}_{6}$ in certain coq6 and coq9 mutants $[18,21,26]$ supplied with $4 \mathrm{HB}$. (B) Hypothetical steps in yeast $\mathrm{CoQ}$ biosynthesis based on the detection of 4- $\mathrm{AP}_{6}$ in certain coq6 and coq9 mutants [18,21,26] supplied with $\mathrm{pABA}$. Abbreviations: 4- $\mathrm{AP}_{6}, 3$-hexaprenyl-4-aminophenol; 4- $\mathrm{HP}_{6}$, 3-hexaprenyl-1,4-benzoquinol; 4- $\mathrm{HP}_{10}$, 3-decaprenyl-1,4-benzoquinol; DBT, 3-decaprenyl-1,4,5-benzenetriol; DDMQ ${ }_{6} \mathrm{H}_{2}$, 3-hexaprenyl-5-methoxy-1,4-benzenediol; $\mathrm{DDMQ}_{10} \mathrm{H}_{2}$, 3-decaprenyl-5-methoxy-1,4-benzenediol; DHB, 3-decaprenyl-4HB; DPP, 3-decaprenylphenol; HAB, 4-amino-3hexaprenylbenzoic acid; HBT, 3-hexaprenyl-1,4,5-benzenetriol; HHB, 3-hexaprenyl-4HB; HPP, 3-hexaprenylphenol; HPAN, 3-hexaprenylaniline. Intermediates denoted with acronyms in brackets represent those that have not yet been experimentally detected in eukaryotic cells.

In addition to the enzymes that catalyze specific steps in the CoQ biosynthetic pathway, there are several other proteins that are necessary for $\mathrm{CoQ}$ synthesis [2,3,7]. In eukaryotes, they include Coq4/COQ4/AtCOQ4, Coq8/ADCK3-4/AtCOQ8, and Coq9/COQ9/AtCOQ9 [2,3,7]. Coq11 is also needed for efficient CoQ biosynthesis in yeast but no mammalian or plant orthologs have been identified to date $[34,35]$. The specific functions of these proteins are still under study. Moreover, it is well established that a large multi-protein complex, termed the CoQ synthome or CoQ complex, is necessary for CoQ biosynthesis [2,36,37]. The proteins Coq3-Coq9 and Coq11 assemble into the CoQ synthome to perform their functions; the elimination/alteration of any one of them is sufficient to destabilize the complex, resulting in impaired CoQ biosynthesis [26,36,37], with the exception of Coq11, the absence of which has been related to a stabilized CoQ synthome [34]. Nevertheless, Coq11 is required for efficient biosynthesis $[34,35]$. Although the majority of the information available about the CoQ synthome is derived from yeast studies, this high molecular weight complex is conserved in higher eukaryotes [38-41]. Recent studies localized the CoQ synthome to specific loci in the mitochondria called $\mathrm{CoQ}$ domains, which are adjacent to ER-mitochondria contact sites [42,43]. There is an additional Coq protein, Coq10/COQ10A-B/AtCOQ10A, which is not essential for CoQ production but it is important in the maintenance of the $\mathrm{CoQ}$ synthome, $\mathrm{CoQ}$ domains, respiratory electron transport, and efficient $\mathrm{CoQ}$ biosynthesis $[2,42-44]$. Similarly, E. coli requires additional proteins for efficient CoQ biosynthesis (UbiB, UbiJ, UbiK, and PasT/RatA) that are not directly involved in the modifications of the ring itself $[2,16,45]$. Of note, $\mathrm{UbiB}$ is the homolog of Coq8 [7], whereas PasT/RatA is the homolog of Coq10 in S. cerevisiae [45]. A recent study identified the existence of a soluble metabolon necessary for $\mathrm{CoQ}$ biosynthesis in bacteria in which the Ubi catalytic enzymes assemble with UbiJ and UbiK to perform their function [16]. 
Understanding the steps involved in CoQ biosynthesis is especially relevant in the context of human disease. Deficiencies in the production of this lipid produce a type of mitochondrial disease known as CoQ deficiencies. Decreased content of CoQ in patients can be caused by direct alteration of the proteins involved in CoQ biosynthesis (primary deficiencies) or by defects not directly linked with CoQ biosynthesis (secondary deficiencies) [46]. Secondary deficiencies can have a genetic origin but they can also result from non-genetic conditions including, clinical treatments (e.g., hypercholesterolemia treatment with statins), environmental toxins, fibromyalgia, metabolic disorders, aging, and agerelated diseases $[3,46-49]$. Primary deficiencies are very rare and affect the central and peripheral nervous system; sensory organs; and heart, muscle, and renal systems $[5,46]$. Secondary $\mathrm{CoQ}$ deficiencies are much more frequent and more heterogeneous, highlighting the diverse functional roles of $\mathrm{CoQ}$ in mitochondrial and non-mitochondrial membranes [46]. CoQ deficiencies can be treated using CoQ supplements, which have been proven to be beneficial for the outcomes of some patients; however, in general, CoQ supplements are inefficient and far from being a reliable therapeutic tool.

For patients suffering from CoQ deficiencies, it is of vital importance to expand our knowledge about how endogenous $\mathrm{CoQ}$ is synthesized, and whether alternative precursors or bypass molecules may provide paths to enhance $\mathrm{CoQ}$ production. It is also important to know how mitochondrially synthesized CoQ is trafficked to other cellular membranes, and how exogenous CoQ is taken up and transported. These topics are the subject of much current research. In this review, we appraise what is known about the origin of the benzenoid ring of CoQ in humans and different model organisms, and highlight how stable isotopes provide a powerful strategy to delineate CoQ biosynthesis.

\section{4-Hydroxybenzoic Acid (4HB)}

\subsection{Classic Ring Precursor Identification}

\subsubsection{Radiolabeling}

Early research on CoQ biosynthesis tested whether ${ }^{14} \mathrm{C}$-radiolabeled precursors could be utilized by cells to generate ${ }^{14} \mathrm{C}-\mathrm{CoQ}$. There were conflicting reports on the ability of a photosynthetic prokaryote, Rhodospirillum rubrum, to incorporate [U- $\left.-{ }^{14} \mathrm{C}\right]$-L-tyrosine into ${ }^{14} \mathrm{C}$-labeled CoQ. These conflicts were resolved by the work of Parson and Rudney [50], who discovered that a trace contaminant present in a commercial source of $\left[\mathrm{U}-{ }^{14} \mathrm{C}\right]-\mathrm{L}-$ tyrosine, $[\mathrm{U}-14 \mathrm{C}]-4$-hydroxybenzaldehyde $(4-\mathrm{Hbz})$, served as a very efficient ring precursor of $\mathrm{CoQ}_{10}$ in Rhodospirillum rubrum. They next showed that both $\left[\mathrm{U}-{ }^{14} \mathrm{C}\right]-4-\mathrm{Hbz}$ and $[\mathrm{U}-14 \mathrm{C}]-4 \mathrm{HB}$ were equally efficient ring precursors of $\mathrm{CoQ}$ biosynthesis in yeast, rats, and Azotobacter vinlandii [51]. Their research set the stage for subsequent metabolic labeling studies, and $\left[\mathrm{U}-{ }^{14} \mathrm{C}\right]-4 \mathrm{HB}$ became the canonical ring precursor for examining CoQ biosynthesis in a wide array of organisms [52].

\subsubsection{Stable Isotope}

Metabolic labeling studies with ${ }^{13} \mathrm{C}_{6}$-ring-labeled $4 \mathrm{HB}$ has become the method of choice to track the biosynthesis of ${ }^{13} \mathrm{C}_{6}$-CoQ and to detect ${ }^{13} \mathrm{C}_{6}$-labeled intermediates. Lipid extracts prepared from cells or from subcellular fractions are separated using HPLC and the ${ }^{13} \mathrm{C}$-ring carbons in both the precursor and product ions can be detected using mass spectrometry (Figure 3). Typically, both normal (unlabeled) and +6 (labeled) precursor ions are detected. The hydroquinone $\left(\mathrm{CoQH}_{2}\right)$ is more polar and elutes earlier via reverse phase chromatography than the quinone $(\mathrm{CoQ})$, and the precursor $\mathrm{CoQH}_{2}$ ion is distinguished by an $m / z$ of +2 relative to the quinone. The characteristic tropylium and chromenylium ions generated by fragmentation of prenylated quinones and prenylated benzenoid rings [53], are also detected as unlabeled and +6 labeled product ions (Figure 3). The tropylium and chromenylium product ions provide information about the ring substituents. In summary, metabolic labeling with ${ }^{13} \mathrm{C}_{6}$-ring-labeled $4 \mathrm{HB}$ provides useful information about the nature of the CoQ intermediates and can also be used to indicate relative rates of de novo biosynthesis. 


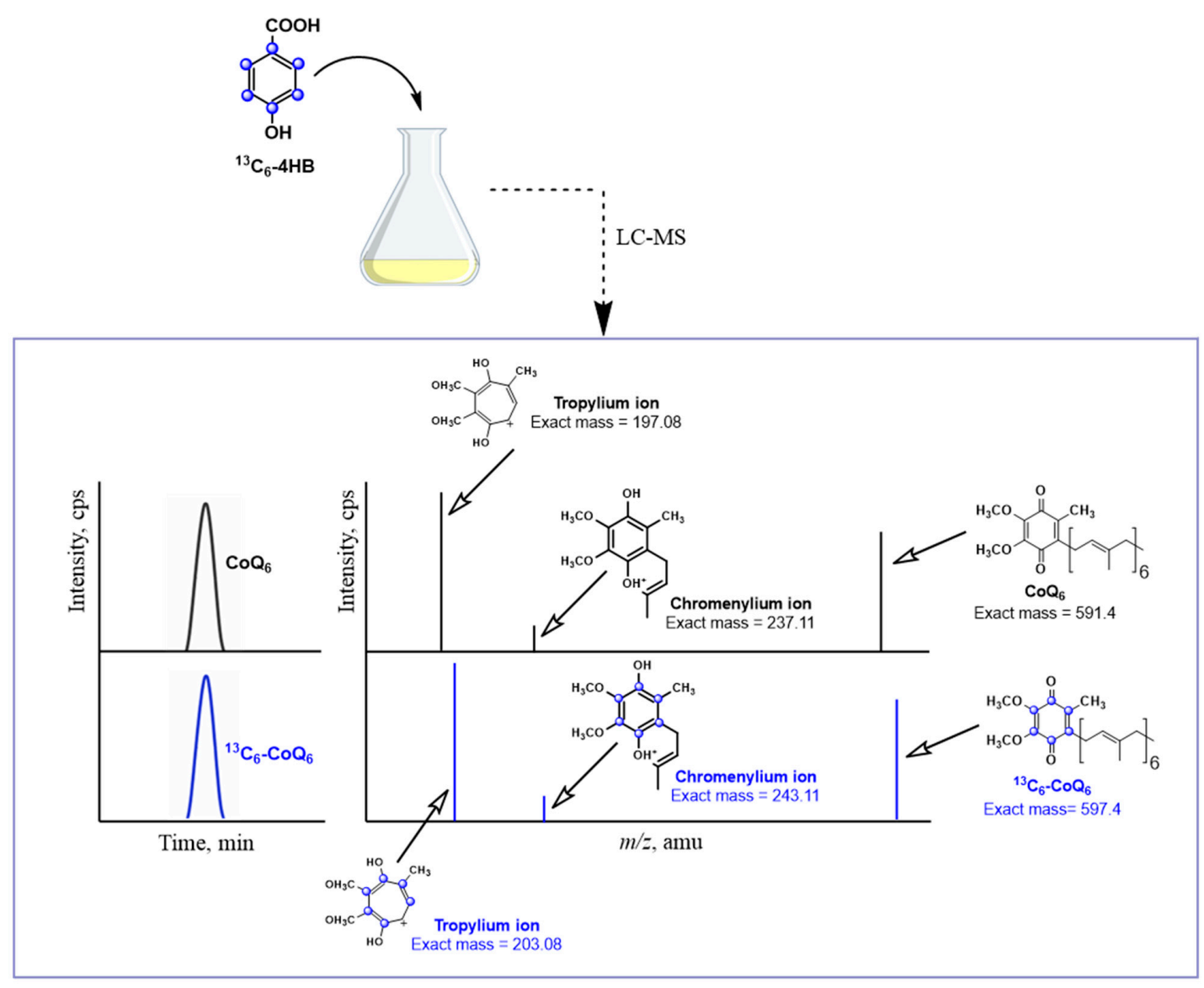

Figure 3. Utilization of stable isotopes and LC-MS to study CoQ biosynthesis. ${ }^{13} \mathrm{C}$-ring-labeled compounds are efficient tools to investigate CoQ biosynthesis. In this illustration, ${ }^{13} \mathrm{C}_{6}$-labeled $4 \mathrm{HB}\left({ }^{13} \mathrm{C}_{6}-4 \mathrm{HB}\right)$ is added to a yeast culture as an example. When cells are treated with ${ }^{13} \mathrm{C}_{6}-4 \mathrm{HB}$, this labeled molecule is incorporated into CoQ biosynthesis, generating ${ }^{13} \mathrm{C}_{6}-\mathrm{CoQ}_{6}$. Analyzing the samples with LC-MS, both non-labeled (endogenous CoQ) and labeled ${ }^{13} \mathrm{C}_{6}$-CoQ (de novo synthesized $\mathrm{CoQ}$ ) can be differentiated by their mass and the mass of their specific tropylium and chromenylium ions. In a similar way, ${ }^{13} \mathrm{C}_{6}$-labeled CoQ intermediates can also be detected and studied. For simplicity, only the oxidized form of $\mathrm{CoQ}_{6}$ is depicted. Product ion spectra are modeled for unlabeled $\mathrm{CoQ}_{6}$ and for the ${ }^{13} \mathrm{C}_{6}-\mathrm{CoQ}_{6}$. $\mathrm{CoQ}_{6}[\mathrm{M}+\mathrm{H}]^{+}$precursor ion $\left(\mathrm{C}_{39} \mathrm{H}_{58} \mathrm{O}_{4}{ }^{+}\right.$, exact mass: 591.4), the $\mathrm{CoQ}_{6}$ tropylium ion $[\mathrm{M}]^{+}\left(\mathrm{C}_{10} \mathrm{H}_{13} \mathrm{O}_{4}{ }^{+}\right.$, exact mass: 197.08), and the CoQ ${ }_{6}$ chromenylium ion $[\mathrm{M}]^{+}\left(\mathrm{C}_{13} \mathrm{H}_{17} \mathrm{O}_{4}{ }^{+}\right.$, exact mass: 237.11); and the ${ }^{13} \mathrm{C}_{6}-\mathrm{CoQ}_{6}[\mathrm{M}+\mathrm{H}]^{+}$precursor ion $\left({ }^{13} \mathrm{C}_{6}{ }^{12} \mathrm{C}_{33} \mathrm{H}_{58} \mathrm{O}_{4}{ }^{+}\right.$, exact mass: 597.4), the ${ }^{13} \mathrm{C}_{6}-\mathrm{CoQ}_{6}$ tropylium ion $[\mathrm{M}]{ }^{+}\left({ }^{13} \mathrm{C}_{6}{ }^{12} \mathrm{C}_{4} \mathrm{H}_{13} \mathrm{O}_{4}{ }^{+}\right.$, exact mass: 203.08), and the ${ }^{13} \mathrm{C}_{6}$-CoQ $\mathrm{Q}_{6}$ chromenylium ion $[\mathrm{M}]^{+}\left({ }^{13} \mathrm{C}_{6}\right.$ $\mathrm{C}_{7} \mathrm{H}_{17} \mathrm{O}_{4}{ }^{+}$, exact mass: 243.11).

\subsection{Source of $4 H B$}

Despite the early recognition that $4 \mathrm{HB}$ served as a universal precursor of CoQ biosynthesis, the pathways leading to its formation in eukaryotes have remained mysterious for decades, and persist as an active topic of research. In animal and human cells, it was recognized that the essential amino acid tyrosine (or phenylalanine via phenylalanine hydroxylase) served as the source of $4 \mathrm{HB}[54,55]$. Although there are still outstanding questions about the steps used to convert tyrosine to $4 \mathrm{HB}$, recent insights into the steps leading from tyrosine to $4 \mathrm{HB}$ have benefitted from the use of stable isotopes. Stable isotopes have also illuminated paths to $4 \mathrm{HB}$ in E. coli, yeast, and plants.

\subsubsection{E. coli}

E. coli produces $4 \mathrm{HB}$ in one step via chorismate pyruvate lyase (Figure 4A) [56]. The authors performed an elegant metabolic labeling experiment with $\left[1,7-{ }^{13} C_{2}\right]$ shikimate, which contains one ${ }^{13} \mathrm{C}$ atom in the carboxyl group and one ${ }^{13} \mathrm{C}$ atom in the adjacent ring 
position. The $\left[1,7-{ }^{13} \mathrm{C}_{2}\right]$ shikimate was fed to an E. coli ubiA mutant that was unable to prenylate $4 \mathrm{HB}$, and the labeled $4 \mathrm{HB}$ that accumulated in the culture medium was recovered and purified. The analysis of the ${ }^{13} \mathrm{C}$-labeled $4 \mathrm{HB}$ by ${ }^{13} \mathrm{C}$ NMR demonstrated that two ${ }^{13} \mathrm{C}$ atoms were retained in the carboxyl group and in the adjacent ring position of $4 \mathrm{HB}$. Moreover, the incorporation of the ${ }^{13} \mathrm{C}$ carboxyl group into $4 \mathrm{HB}$ from shikimate proceeded with greater than $99 \%$ retention, indicating that under the labeling conditions employed, the production of $4 \mathrm{HB}$ in E. coli was due exclusively to the reaction catalyzed by UbiC.

However, it is possible that another pathway to $4 \mathrm{HB}$ exists in $E$. coli, because $u b i C$ mutants still synthesize $\mathrm{CoQ}_{8}[56,57]$. Although it was thought that the original $u b i C$ mutant isolated and characterized might be leaky, the persistence of CoQ synthesis was also observed in a mutant derived from a ubiC single-gene knockout mutant of the Keio collection [27]. Instead, it is generally considered that the leaky phenotype of $u b i C$ mutants may be due to the chemical decomposition of chorismate to $4 \mathrm{HB}$ [56]. It is also possible that under certain conditions, E. coli cells may make use of other ring precursors (see Section 3.2).
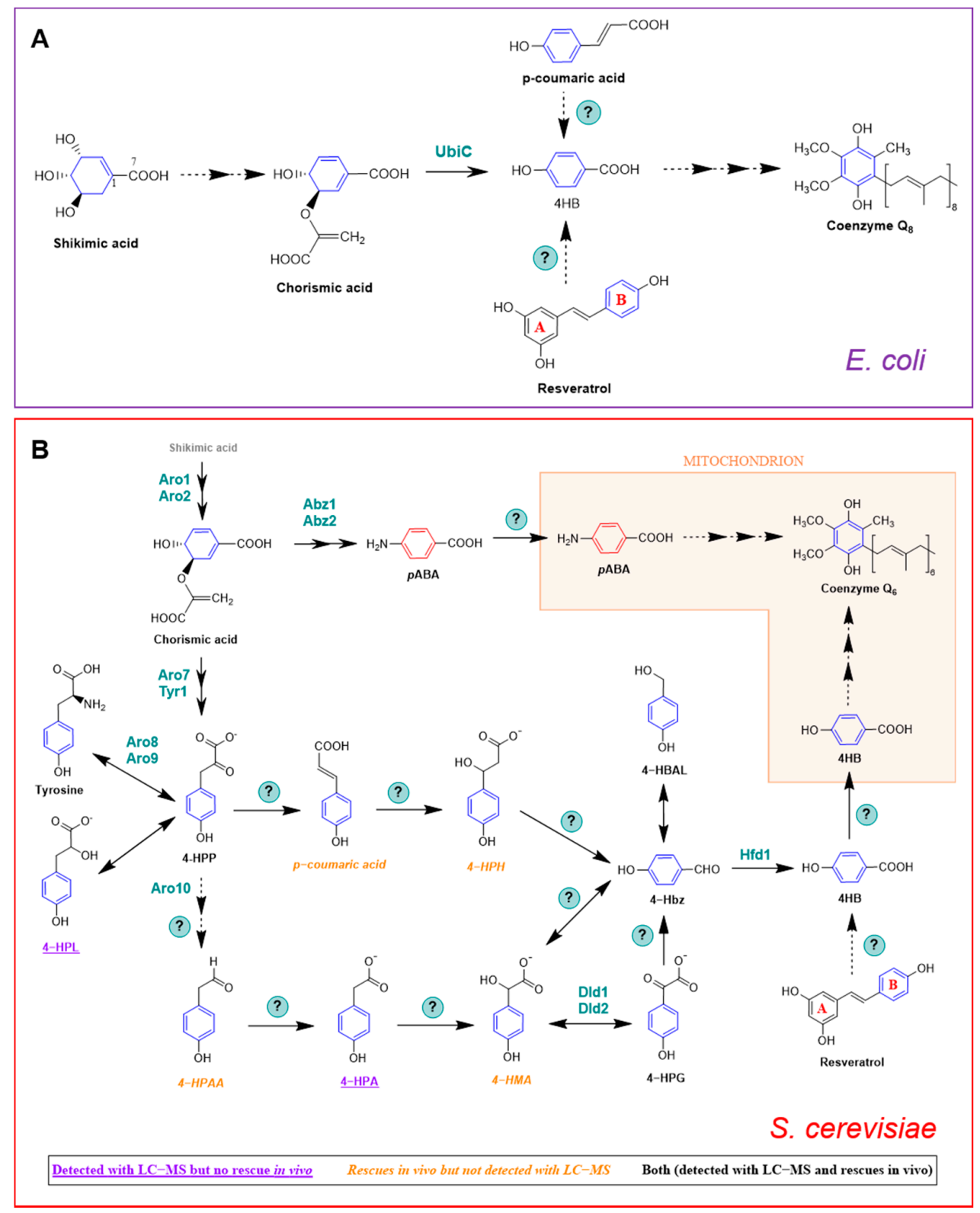

Figure 4. CoQ ring precursors in E. coli and S. cerevisiae. (A) $4 \mathrm{HB}$ is the main CoQ precursor in E. coli and it is derived from chorismic acid, but $p$-coumaric acid and resveratrol can additionally act as ring precursors. Uniquely, CoQ biosynthesis in bacteria is thought to occur in the cytosol [16]. (B) 4HB, 
pABA, $p$-coumaric acid, and resveratrol can serve as CoQ ring precursors in S. cerevisiae. The diagram unifies what is described in $[17,18,27,58-62]$. Catalytic enzymes are named in their specific steps. Steps labeled with a (?) indicate that the responsible enzyme(s) is unknown. Abbreviations: 4-HBAL, 4-hydroxybenzylalcohol; 4-Hbz, 4-hydroxybenzaldehide; 4-HMA, 4-hydroxymandelic acid; 4-HPA, 4-hydroxyphenylacetate; 4-HPAA, 4-hydroxyphenylacetaldehyde; 4-HPG, 4hydroxyphenylglyoxylate; 4-HPH, 4-hydroxyphenylhydracrylic acid; 4-HPL, 4-hydroxyphenyllactate; 4-HPP, 4-hydroxyphenylpyruvic acid. Figure modified from Robinson et al. [62].

\subsubsection{S. cerevisiae}

Yeast synthesizes $4 \mathrm{HB}$ via a pathway that is distinct from that of E. coli. S. cerevisiae can also produce $4 \mathrm{HB}$ via the shikimate pathway; however, no homolog of $u b i \mathrm{C}$ exists in the $S$. cerevisiae genome. Although many questions persist, stable isotopes have helped to delineate this pathway. Payet et al. [58] showed that ${ }^{15} \mathrm{~N}^{13} \mathrm{C}_{9}$-tyrosine produced a robust labeling of ${ }^{13} \mathrm{C}_{6}$-CoQ, whereas analogously labeled phenylalanine failed to be incorporated into $\mathrm{CoQ}$ by $S$. cerevisiae. Yeast lacks phenylalanine hydroxylase, indicating that there is no independent pathway from phenylalanine to $4 \mathrm{HB}$. Payet et al. [58] also demonstrated that the Aro8 and Aro9 aminotransferases that interconvert tyrosine and 4-hydroxyphenylpyruvate (4-HPP) constitute an important step in the pathway to $4 \mathrm{HB}$. As shown in Figure 4B, 4-HPP may be produced from either the deamination of tyrosine, or in two steps from chorismate. Payet et al. [58] then performed a forward genetic screen in yeast aro 2 mutants, and selected for mutants that retained the ability to grow on a nonfermentable medium, provided that $4 \mathrm{HB}$ was added. This screen identified the $h f d 1$ mutant, and subsequent deletion of $h f d 1$ in a wild-type yeast background resulted in a CoQ deficiency [58]. The authors then showed that the Hfd1-dependent oxidation of 4hydroxybenzaldehyde $(4-\mathrm{Hbz})$ to $4 \mathrm{HB}$ is an essential step in the production of $4 \mathrm{HB}$ from tyrosine. Addition of ${ }^{15} \mathrm{~N}^{13} \mathrm{C}_{9}$-tyrosine produced $\mathrm{a}+7 \mathrm{~m} / \mathrm{z}$ in $4-\mathrm{Hbz}$ in both wild-type and $h f d 1$ mutant yeast. Expression of ALDH3A1, a human homolog of yeast Hfd1, restored the ability of the $h f d 1$ mutant to synthesize CoQ, and also increased the CoQ content when $4-\mathrm{Hbz}$ was added to the culture medium [58]. This study characterized the first and last intermediates of the pathway from tyrosine to $4 \mathrm{HB}$.

In a completely independent study, Stefely et al. [59] used mass spectrometry to map the proteomes, lipidomes, and metabolomes of a collection of $S$. cerevisiae gene deletion mutants, chosen because each deleted gene encoded an uncharacterized mitochondrial protein. Stefely et al. [59] generated an enormous and intriguing set of data. The authors focused their follow up biochemical studies on the $h f d 1$ null mutant because it showed decreased contents of $4 \mathrm{HB}$ and $\mathrm{HHB}$, which are early CoQ intermediates (Figure 1A) [59]. Despite the deficiencies in these two early intermediates of CoQ biosynthesis, the CoQ content in the $h f d 1$ mutant was normal. This is because $S$. cerevisiae can utilize $p$ ABA to synthesize CoQ (see Section 3.1). It is important to note that the aforementioned study conducted by Payet et al. [58] was performed in medium that lacked $p$ ABA. Stefely et al. [59] showed that Hfd1 functions as an aldehyde dehydrogenase, and performs the final step in $4 \mathrm{HB}$ biosynthesis. They also showed that the expression of human ALDH3A1 complements the yeast $h f d 1$ mutant, suggesting that it plays a similar role in human cells. Thus, these two studies used entirely different approaches, and both shed light on the path to 4HB [63].

Recent work by Valera et al. $[60,61]$ has suggested possible intermediates in the conversion of 4-hydroxyphenylpyruvate (4-HPP) to 4-hydroxybenzaldehyde (4-Hbz) (Figure 4B). The authors hypothesized that the mandelate pathway may provide a conduit between 4-HPP and 4-Hbz in yeast. They took advantage of the yeast Hanseniaspora vineae, which produces 100 times more phenylpropanoid compounds than S. cerevisiae. The production of $4-\mathrm{Hbz}$ in $\mathrm{H}$. vineae requires the thiamine diphosphate-dependent decarboxylation of 4-hydroxyphenylglyoxylate (4-HPG). Treatment of $H$. vineae with ${ }^{13} \mathrm{C}$-tyrosine and methylbenzylphosphonate, an inhibitor of benzylformate decarboxylase, decreased the production of ${ }^{13} \mathrm{C}$-labeled 4 - $\mathrm{Hbz}$ and resulted in the accumulation of several ${ }^{13} \mathrm{C}$-labeled metabolites characteristic of the mandelate pathway, including ${ }^{13} \mathrm{C}-4$-hydroxymandelate (4-HMA) [60]. 
The authors then turned to $S$. cerevisiae and showed that mutants harboring deletions in ARO10 failed to produce 4- $\mathrm{Hbz}[60,61]$. Yeast mutants harboring deletions in either DLD1 or DLD2 showed five- and ten-times decreased production of 4-Hbz, respectively [60]. This work suggests that the mandelate pathway may be responsible for producing the $4 \mathrm{HB}$ ring precursor of $\mathrm{CoQ}$ biosynthesis, and that Aro10 was postulated to perform two decarboxylation steps. However, the role of this pathway in S. cerevisiae CoQ biosynthesis needs to be demonstrated by ${ }^{13} \mathrm{C}$-metabolic tracing experiments.

A recent study by Robinson et al. [62] indicates that study of the metabolism between $4-\mathrm{HPP}$ and $4-\mathrm{Hbz}$ is quite challenging, and the exact role of Aro10, and the order of intermediates in the pathway is still quite speculative (Figure 4B). Robinson et al. [62] uncovered layers of redundancy, and showed that readily identified metabolites may be unproductive, whereas in contrast, productive metabolites may be fleeting in nature and extremely difficult to detect. They identified three aminotransferases (Bat2, Bna3, and Aat2) that are able to produce sufficient 4-HPP to support $4 \mathrm{HB}$ biosynthesis from tyrosine in the absence of Aro2, Aro8, and Aro9. Unexpectedly, deletion of all six genes (aro2 plus each of the five aminotransferases) generates a yeast mutant that shows low respiration even in the presence of added $4 \mathrm{HB}$. The authors noted that the absence of one of the aminotransferases (Aat2) impaired respiratory growth for unknown reasons. The authors then tested whether deletion of HFD1 would cause ${ }^{13} \mathrm{C}$-labeled tyrosine metabolites to accumulate. They observed that while 4-HPL accumulated, 4-HPP, 4-HPA, and 4- $\mathrm{Hbz}$ were all decreased in the $h f d 1$ mutant as compared to wild type. They speculated that these decreases could be due to the loss of Hfd1, or might result from a general respiratory deficiency. In the latter case, Robinson et al. [62] postulated that an increase in the ratio of 4-HPL to 4-HPP may reflect the need for $\mathrm{NAD}^{+}$, and their interconversion may function similarly to lactate/pyruvate, to maintain the $\mathrm{NAD}^{+} / \mathrm{NADH}$ redox balance necessary for growth [64]. The other confounding observation was that the most abundant ${ }^{13} \mathrm{C}$-labeled catabolites (4-HPA and 4-HPL) did not rescue the respiratory growth deficiency when added to the aro8aro9aro 2 mutant. In contrast, the compounds that rescued the best (4HPAA and 4-HMA) were not observed as ${ }^{13} \mathrm{C}$-labeled catabolites in S. cerevisiae. Finally, the authors showed that Aro10 is dispensable for the synthesis of $4 \mathrm{HB}$. The overall conclusion is that the pathway from tyrosine to $4-\mathrm{Hbz}$ is incredibly robust, and that further work with ${ }^{13} \mathrm{C}$-labeled intermediates will be required to delineate the pathway(s) from 4-HPP to $4-\mathrm{Hbz}$.

\subsubsection{Mammals}

Mammals lack the shikimate pathway and so $4 \mathrm{HB}$ must be derived from a different precursor. Early studies on $\mathrm{CoQ}$ research using radiolabeled compounds demonstrated that the origins of the aromatic ring in animal cells are the essential amino acids phenylalanine and tyrosine $[52,65]$ (Figure $5 \mathrm{~A}$ ), and that $\left[\mathrm{U}-{ }^{14} \mathrm{C}\right]$ tyrosine is a 3 -fold better precursor than $\left[\mathrm{U}-{ }^{14} \mathrm{C}\right]$ phenylalanine in rat liver slices [54]. In fact, the incorporation of phenylalanine into $4 \mathrm{HB}$ is made after its conversion to tyrosine by a phenylalanine hydroxylase (PAH) [7] (Figure 5A). Tyrosine aminotransferase (TAT) catalyzes the transamination of tyrosine into 4-HPP [66]. Additionally, the mammalian homolog of the yeast Aro8/Aro9, AADAT, is also a candidate to carry out this reaction [7] (Figure 5A). Very little is known about the multiple steps occurring between 4-HPP and 4HB and all the information available to date is derived from yeast studies [58-62] (see Section 2.2.2). ALDH3A1, the human homolog of the yeast $\mathrm{Hfd} 1$, restored the oxidation of $4 \mathrm{Hbz}$ to $4 \mathrm{HB}$ when expressed in the yeast $h f d 1$ mutant. This suggests that at least the last step from tyrosine to $4 \mathrm{HB}$ is conserved from yeast to humans $[58,59,63]$. 

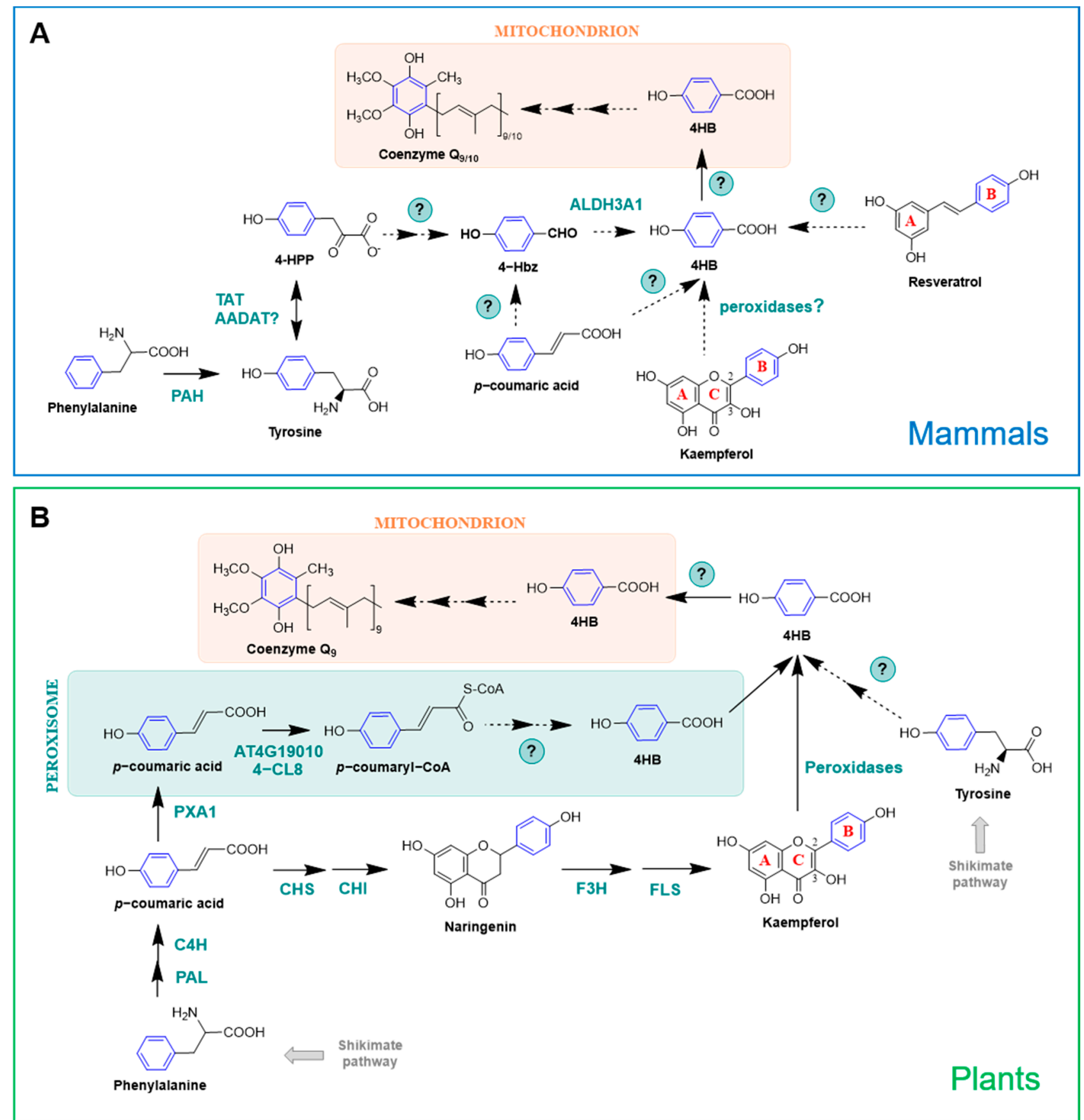

Figure 5. CoQ ring precursors in mammals and plants. (A) In mammals, $4 \mathrm{HB}$ is derived from dietary tyrosine and phenylalanine. $p$-coumaric acid, resveratrol, and kaempferol can additionally serve as CoQ ring precursors. (B) Plants derive $4 \mathrm{HB}$ from tyrosine and phenylalanine via a non-intersecting pathway. In fact, plants can derive $4 \mathrm{HB}$ from phenylalanine by two parallel branches. One of them occurs in the cytosol and is linked to the biosynthesis pathway of the flavonol kaempferol, and the other one involves peroxisomes and $\beta$-oxidation [67]. Catalytic enzymes are named in their specific steps. Steps labeled with a (?) indicate that the responsible enzyme(s) is unknown. Abbreviations in panels (A,B): 4-Hbz, 4-hydroxybenzaldehide; 4-HPP, 4-hydroxyphenylpyruvic acid; 4-CL8, 4-coumarate-CoA ligase 8; AADAT, mitochondrial alpha-aminoadipate aminotransferase; ALDH3A1, aldehyde dehydrogenase 3A1; AT4G19010, peroxisomal p-coumaroyl-CoA ligase; $\mathrm{C} 4 \mathrm{H}$, cinnamate-4-hydroxylase; $\mathrm{CHS}$, chalcone synthase; $\mathrm{CHI}$, chalcone isomerase; $\mathrm{F} 3 \mathrm{H}$, flavanone-3-hydroxylase; FLS, flavonol synthase; PAH, phenylalanine hydrolase; PAL, phenylalanine ammonia lyase; PXA1, peroxisomal $\mathrm{ABC}$ transporter 1. 


\subsubsection{Plants}

In plants, $4 \mathrm{HB}$ is also derived from phenylalanine and tyrosine (Figure 5B). However, unlike mammals, they use two nonintersecting routes for this purpose, since Arabidopsis mutants unable to utilize phenylalanine were still able to utilize tyrosine as a ring precursor of $\mathrm{CoQ}_{9}$ [68]. Studies involving stable isotopes determined that plants have the unique ability to synthesize $4 \mathrm{HB}$ from the $\beta$-oxidative metabolism of phenylalanine, which is actually the preferred substrate for CoQ biosynthesis in Arabidopsis, supplying approximately $70 \%-80 \%$ of the CoQ biosynthetic flux $[67,68]$. In this pathway, phenylalanine is deaminated and hydroxylated, producing $p$-coumarate in the cytosol. These reactions are common to all land plants as part of the phenylpropanoid pathway and are catalyzed, respectively, by phenylalanine lyase (PAL) and cinnamate-4-hydroxylase $(\mathrm{C} 4 \mathrm{H})[68,69]$. Then, $p$-coumarate is imported into peroxisomes and transformed in $p$-coumaryl-CoA by a $p$-coumarate-CoA ligase (At4g19010 in Arabidopsis thaliana). Subsequent steps of hydration, oxidation, thiolation, and $\mathrm{CoA}$ thioester hydrolysis complete the $\beta$-oxidation of $p$-coumaroyl-CoA into $4 \mathrm{HB}[68,69]$. The enzymes responsible for catalyzing these reactions are still unknown, but have been proposed to be similar to the ones involved in the conversion of cinnamoyl-CoA into benzoate [69]. In a recent study, isotopic feeding assays, gene co-expression analysis, and reverse genetics demonstrated that Arabidopsis possesses an additional $p$-coumaroyl-CoA ligase (named 4-coumarate-CoA ligase 8, 4-CL8; At5g38120) that participates in the $\beta$-oxidative metabolism of $p$-coumarate to produce $4 \mathrm{HB}$ in peroxisomes [67]. Both 4-CL8 and At4g19010 belong to the same clade (V) of acyl-activating enzymes, but phylogenetic analysis indicated that they are not paralogous [67].

Plants are unique in their ability to synthesize $4 \mathrm{HB}$ from phenylpropanoids in a pathway that uses phenylalanine as a precursor but is $\beta$-oxidation-independent and occurs outside peroxisomes [69]. In fact, the import of $p$-coumarate into peroxisomes creates the split between these two branches. In the cytosol, $p$-coumarate enters the biosynthesis of flavonoids, eventually forming the hydroxyphenyl moiety (i.e., B-ring) of kaempferol, which is further cleaved to produce $4 \mathrm{HB}[67,69]$ (Figure 5B). This cytosolic route of $4 \mathrm{HB}$ formation contributes to $\sim 20 \%$ of $\mathrm{CoQ}$ formation in Arabidopsis [69]. Kaempferol utilization for $\mathrm{CoQ}$ biosynthesis in plants and other organisms is explored further in Section 3.2.

\section{Other Ring Precursors}

\section{1. $p A B A$ in S. cerevisiae}

A role for $\mathrm{pABA}$ as a ring precursor in CoQ biosynthesis was discovered in 2010 by two independent research groups $[17,18]$. This result was surprising for two reasons: (1) pABA was generally considered a dedicated ring precursor in folate biosynthesis; and (2) there is no nitrogen substituent on the ring of CoQ. Pierrel et al. [18] were probing the role of mitochondrial ferredoxin (Yah1) and ferredoxin reductase (Arh1) in the hydroxylase steps of $\mathrm{CoQ}_{6}$ biosynthesis. They discovered that cells with deficiencies in either Yah1 or Arh1 accumulated the intermediates 3-hexaprenyl-4-hydroxyphenol (4- $\mathrm{HP}_{6}$; analogous to $4-\mathrm{HP}_{10}$ in Figure 2A) and 3-hexaprenyl-4-aminophenol (4- $\mathrm{AP}_{6}$, Figure $\left.2 \mathrm{~B}\right)$. The presence of the nitrogen atom in the ring of this latter metabolite suggested that the ring precursor might be pABA. Feeding yeast cultures with $\mathrm{U}^{13} \mathrm{C}$-pABA produced $+6 \mathrm{~m} / \mathrm{z}^{13} \mathrm{C}$-ring-labeled $\mathrm{CoQ}_{6}$ in wild-type cells, as well as the corresponding $+6 \mathrm{~m} / z$ ion of 3-hexaprenyl-4-aminophenol in the Yah1-deficient cells. In addition, it was shown that pABA and $4 \mathrm{HB}$ compete for the Coq2-mediated prenylation step, and the early intermediate hexaprenyl-aminobenzoic acid (HAB) was detected in coq null mutants known to accumulate HHB (Figure 1A).

HAB was independently discovered by Marbois et al. [17] as a naturally occurring lipid component of yeast cells cultured in rich medium. HAB was originally detected by screening for product ions (such as 95 and 109), characteristically produced via the fragmentation of prenylated molecules. The elution of HAB was resolved by RP-HPLC from that of HHB by $0.2 \mathrm{~min}$, and the precursor ion and the predominant tropylium and chromenylium product ions, detected in ion-trap analyses, were $1 \mathrm{amu}$ less than expected for HHB. The difference of one mass unit was consistent with an amino substituent 
replacing the hydroxyl substituent. ${ }^{13} \mathrm{C}_{6}-\mathrm{HAB},{ }^{13} \mathrm{C}_{6}-\mathrm{DMQ}_{6}$, and ${ }^{13} \mathrm{C}_{6}-\mathrm{CoQ}_{6}$ were produced in wild-type yeast cultured with ${ }^{13} \mathrm{C}_{6}$-pABA. Yeast abz1 mutants cultured under conditions where exogenous pABA was eliminated continued to synthesize $\mathrm{CoQ}_{6}$, but $\mathrm{HAB}$ could no longer be detected. The production of ${ }^{13} \mathrm{C}_{6}-\mathrm{HAB}$ was eliminated in a coq 2 mutant. Retention of the amino substituent was also detected in $\mathrm{IDMQ}_{6}$ (Figure 1). However, this metabolite is now considered to be a dead-end intermediate, since it was shown that Coq6 conducts the oxidative removal of the amino group [20]. The ability of $S$. pombe to use $p A B A$ to synthesize $\mathrm{CoQ}_{10}$ was recently described [19].

\subsection{Natural Products}

Phenolic compounds are secondary metabolites in plants that possess an aromatic ring bearing one or more hydroxyl groups. They can be classified into simple and complex phenolics (or polyphenols). Simple phenolics contain a carboxylic group on the benzene ring with one or more hydroxyl or methoxyl substituents, whereas polyphenols contain more than one benzene ring [70,71]. Using a more detailed classification method, phenolic compounds are divided into five major chemical families: flavonoids, phenolic acids, stilbenes, lignans, and curcuminoids [72]. These compounds are present in a wide array of foods and beverages of plant origin, and have received great interest due to their positive effects on human health. A growing body of evidence suggests a role for polyphenols in the prevention of important diseases, including cancer, chronic inflammation, as well as cardiovascular and neurodegenerative diseases [73]. The beneficial properties of polyphenols have been partially attributed to their antioxidant role, and to their ability to modulate molecular targets and signaling pathways [71,73].

Shortly after the identification of $p$-coumarate as a CoQ ring precursor in plants [68], its fate in other organisms was studied [27]. Using ${ }^{13} \mathrm{C}_{6}$-coumarate, authors described that CoQ can be derived from $p$-coumarate in E. coli, S. cerevisiae, and mammalian cells [27] (Figures 4 and $5 \mathrm{~A}$ ). Given the structural similarity of resveratrol with $p$-coumarate, the incorporation of ${ }^{13} \mathrm{C}_{6}$-resveratrol into ${ }^{13} \mathrm{C}_{6}$-CoQ was additionally explored, showing that resveratrol serves as an aromatic ring precursor in CoQ biosynthesis in E. coli, yeast, and mammalian cells [27] (Figures 4 and 5A). Wild-type E. coli cells barely incorporated ${ }^{13} \mathrm{C}_{6}$ resveratrol or ${ }^{13} \mathrm{C}_{6}$-coumarate into $\mathrm{CoQ}_{8}$; however, when defects in $4 \mathrm{HB}$ synthesis are present (e.g., in E. coli ubiC mutants), the incorporation of resveratrol and $p$-coumarate was dramatically enhanced [27]. In contrast, approximately $10 \%$ of the total CoQ present in mouse and humans cells was derived from ${ }^{13} \mathrm{C}_{6}$-resveratrol after $24 \mathrm{~h}$ [27]. Resveratrol is a stilbene that has been studied extensively due to its purported antiplatelet, antioxidant, anti-inflammatory, blood glucose-lowering, cardiovascular protective, and anti-cancer activities (reviewed in [74]), but that was the first time that a polyphenol was described to serve as precursor for the synthesis of CoQ. This finding also highlighted the fact that exogenous antioxidants can be utilized to synthesize a wholly different class of molecule, CoQ in this case, which could be implicated in the ultimate effects described for the initial compound [27]. The exact mechanism by which resveratrol is incorporated into CoQ biosynthesis is still unknown, although its breakdown inside the cells to yield CoQ ring precursors, such as $4 \mathrm{HB}$, is a possibility.

In a subsequent study, the effect of several polyphenols on CoQ content and biosynthesis was studied [75]. Dose-response analysis showed that resveratrol, apigenin, and kaempferol were able to increase the total CoQ content in renal cells, whereas piceatannol, quercetin, luteolin, and naringenin did not produce any effect [75]. The finding that dietary compounds can enhance the endogenous CoQ content is especially relevant in the research of alternative strategies to palliate CoQ deficiencies $[2,7,46]$. The ability of the flavonol-type flavonoid kaempferol to increase CoQ levels was dramatically superior to the one exerted by the flavone-type flavonoid apigenin or the stilbene resveratrol. Further experiments with ${ }^{13} \mathrm{C}$-kaempferol revealed that kaempferol behaves as a $\mathrm{CoQ}$ ring precursor in mammalian cells [75] (Figure 5A). Similar experiments carried out in S. cerevisiae demonstrated that kaempferol did not increase CoQ content and only marginally entered 
into CoQ biosynthesis, suggesting that yeast cannot utilize flavonoids in the same manner as mammalian cells [75]. ${ }^{13} \mathrm{C}_{12}$-curcumin and $\mathrm{D}_{3}$-ferulic acid were also explored in this study as potential precursors of $\mathrm{CoQ}$ but no isotope-labeled $\mathrm{CoQ}$ was detected, indicating that these polyphenols do not have the ability to serve as CoQ ring precursors [75]. Altogether, these results suggested that the chemical structure is a key factor that defines the functions and the effects of different polyphenols. Flavonoids were observed to be more efficiently used in $\mathrm{CoQ}$ biosynthesis than stilbenoids or curcuminoids. Kaempferol was the one with the strongest effect on increasing CoQ content, highlighting the importance of the hydroxyl group in the $\mathrm{C} 3$ position, which has been previously linked with high antioxidant activity [76], but also the relevance of having only one -OH in the B-ring [75].

The metabolism of kaempferol responsible for its incorporation into CoQ, as well as the part of the molecule entering the biosynthetic pathway, remained unknown until a recent study carried out in plants shed some light onto this topic [69]. In this study, Soubeyrand et al. [69] discovered that there is in fact a direct connection between the biosynthesis of $\mathrm{CoQ}$ and that of flavonoids in Arabidopsis. Using stable isotope feeding assays, and particularly kaempferol that was exclusively labeled in the B-ring $\left({ }^{13} \mathrm{C}-\mathrm{B}\right.$ ring-kaempferol), they demonstrated that Arabidopsis and tomato plants can derive $4 \mathrm{HB}$ specifically from the B-ring of kaempferol, and that such cleavage is catalyzed by hemedependent peroxidases [69] (Figure 5B). In contrast, kaempferol 3- $\beta$-D-glucopyranoside, dihydrokaempferol, and naringenin were resistant to peroxidative cleavage, highlighting that the reaction requires a hydroxyl group on C-3 as well as a double bond between C-2 and C-3 [69], which supports the previous observations made in kidney-derived cells [75]. The glycosylation of kaempferol on its C-3 hydroxyl is believed to prevent the oxidative release of the B-ring as $4 \mathrm{HB}$ and thus its incorporation into CoQ biosynthesis [69]. Supporting this model, new experiments using a complex Arabidopsis mutant that lacks the majority of kaempferol 3-O-glycosyltransferase activities, leaving the C-3 hydroxyl of kaempferol unprotected, showed that the release of the B-ring of kaempferol as $4 \mathrm{HB}$ is increased, boosting CoQ biosynthesis [77]. Additional studies in mammalian cells using ${ }^{13} \mathrm{C}$-B-ring-kaempferol demonstrated that the B-ring is indeed the part of the molecule that is incorporated into $\mathrm{CoQ}$ [78], suggesting that the mechanism described in plants is likely to be conserved in vertebrates. The specific peroxidases in charge of the cleavage of kaempferol into $4 \mathrm{HB}$ still need to be identified, but they are hypothesized to be conserved between plants and mammalian cells and absent in S. cerevisiae, since yeast is described to incorporate ${ }^{13} \mathrm{C}$-kaempferol very marginally $[75,78]$.

Additional studies involving isotope-labeled compounds will help to unravel the specifics of the incorporation of certain flavonoids in the CoQ biosynthetic pathway, as well as to identify new natural compounds that can behave as CoQ ring precursors.

\section{Closing Remarks}

This review summarizes the current knowledge about the origin of the benzenoid ring of CoQ in E. coli, S. cerevisiae, mammals, and plants, and highlights the exceptional contribution that stable isotopes provided to delineating the pathways that lead to the biosynthesis of CoQ. Stable isotopes will be useful to fill the gaps in the pathways described, as well as to explore other molecules as alternative ring precursors for $\mathrm{CoQ}$ biosynthesis. Moreover, stable isotopes can be of great value in the study of CoQ biosynthesis in other contexts, including metabolic and mitochondrial diseases, responses to drugs, or genetic alterations.

Author Contributions: C.F.C. conceptualized the review. L.F.-d.-R. and C.F.C. wrote, edited, revised for intellectual content, and approved the final version of the review. L.F.-d.-R. prepared the figures. All authors have read and agreed to the published version of the manuscript.

Funding: This work was supported by the National Science Foundation Grant MCB-1330803 to C.F.C.

Acknowledgments: We thank the UCLA Molecular Instrumentation Core for the use of the QTRAP 4000. 
Conflicts of Interest: The authors declare no conflict of interest.

\section{References}

1. Aussel, L.; Pierrel, F.; Loiseau, L.; Lombard, M.; Fontecave, M.; Barras, F. Biosynthesis and physiology of coenzyme Q in bacteria. Biochim. Biophys. Acta (BBA)-Bioenerg. 2014, 1837, 1004-1011. [CrossRef]

2. Wang, Y.; Hekimi, S. The Complexity of Making Ubiquinone. Trends Endocrinol. Metab. 2019, 30, 929-943. [CrossRef]

3. Awad, A.M.; Bradley, M.C.; Fernández-Del-Río, L.; Nag, A.; Tsui, H.S.; Clarke, C.F. Coenzyme Q10 deficiencies: Pathways in yeast and humans. Essays Biochem. 2018, 62, 361-376. [CrossRef] [PubMed]

4. Turunen, M.; Olsson, J.; Dallner, G. Metabolism and function of coenzyme Q. Biochim. Biophys. Acta (BBA)-Bioenerg. 2004, 1660, 171-199. [CrossRef]

5. Alcázar-Fabra, M.; Rodríguez-Sánchez, F.; Trevisson, E.; Brea-Calvo, G. Primary coenzyme Q deficiencies: A literature review and online platform of clinical features to uncover genotype-phenotype correlations. Free Radic. Biol. Med. 2021, 167, 141-180. [CrossRef] [PubMed]

6. Wang, Y.; Hekimi, S. Understanding Ubiquinone. Trends Cell Biol. 2016, 26, 367-378. [CrossRef]

7. Stefely, J.A.; Pagliarini, D.J. Biochemistry of Mitochondrial Coenzyme Q Biosynthesis. Trends Biochem. Sci. 2017, 42, 824-843. [CrossRef]

8. Lombard, J.; Moreira, D. Origins and Early Evolution of the Mevalonate Pathway of Isoprenoid Biosynthesis in the Three Domains of Life. Mol. Biol. Evol. 2011, 28, 87-99. [CrossRef] [PubMed]

9. Boronat, A.; Rodríguez-Concepción, M. Terpenoid biosynthesis in prokaryotes. In Biotechnology of Isoprenoids; Schrader, J., Bohlmann, J., Eds.; Advances in Biochemical Engineering/Biotechnology; Springer Nature: Basingstoke, UK, 2015; Volume 148, pp. 3-18.

10. Lohr, M.; Schwender, J.; Polle, J.E. Isoprenoid biosynthesis in eukaryotic phototrophs: A spotlight on algae. Plant Sci. 2012, 185-186, 9-22. [CrossRef] [PubMed]

11. Bentlage, B.; Rogers, T.S.; Bachvaroff, T.R.; Delwiche, C.F. Complex Ancestries of Isoprenoid Synthesis in Dinoflagellates. J. Eukaryot. Microbiol. 2016, 63, 123-137. [CrossRef]

12. Abby, S.S.; Kazemzadeh, K.; Vragniau, C.; Pelosi, L.; Pierrel, F. Advances in bacterial pathways for the biosynthesis of ubiqui-none. Biochim. Biophys. Acta (BBA)-Bioenerg. 2020, 1861, 148259. [CrossRef]

13. Hayashi, K.; Ogiyama, Y.; Yokomi, K.; Nakagawa, T.; Kaino, T.; Kawamukai, M. Functional conservation of coenzyme Q bio-synthetic genes among yeasts, plants, and humans. PLoS ONE 2014, 9, e99038. [CrossRef]

14. Kawamukai, M. Biosynthesis of coenzyme Q in eukaryotes. Biosci. Biotechnol. Biochem. 2016, 80, 23-33. [CrossRef] [PubMed]

15. Tsui, H.S.; Clarke, C.F. Ubiquinone Biosynthetic Complexes in Prokaryotes and Eukaryotes. Cell Chem. Biol. 2019, $26,465-467$. [CrossRef] [PubMed]

16. Chehade, M.H.; Pelosi, L.; Fyfe, C.D.; Loiseau, L.; Rascalou, B.; Brugière, S.; Kazemzadeh, K.; Ciccone, L.; Aussel, L.; Couté, Y.; et al. A Soluble Metabolon Synthesizes the Isoprenoid Lipid Ubiquinone. Cell Chem. Biol. 2019, 26, 482-492. [CrossRef] [PubMed]

17. Marbois, B.; Xie, L.X.; Choi, S.; Hirano, K.; Hyman, K.; Clarke, C.F. para-Aminobenzoic acid is a precursor in coenzyme Q6 bio-synthesis in Saccharomyces cerevisiae. J. Biol. Chem. 2010, 285, 27827-27838. [CrossRef]

18. Pierrel, F.; Hamelin, O.; Douki, T.; Kieffer-Jaquinod, S.; Mühlenhoff, U.; Ozeir, M.; Lill, R.; Fontecave, M. Involvement of mito-chondrial ferredoxin and para-aminobenzoic acid in yeast coenzyme Q biosynthesis. Chem. Biol. 2010, 17, 449-459. [CrossRef]

19. Nishida, I.; Yanai, R.; Matsuo, Y.; Kaino, T.; Kawamukai, M. Benzoic acid inhibits Coenzyme Q biosynthesis in Schizosaccharomyces pombe. PLoS ONE 2020, 15, e0242616. [CrossRef] [PubMed]

20. Ozeir, M.; Mühlenhoff, U.; Webert, H.; Lill, R.; Fontecave, M.; Pierrel, F. Coenzyme Q Biosynthesis: Coq6 Is Required for the C5-Hydroxylation Reaction and Substrate Analogs Rescue Coq6 Deficiency. Chem. Biol. 2011, 18, 1134-1142. [CrossRef]

21. Ozeir, M.; Pelosi, L.; Ismail, A.; Mellot-Draznieks, C.; Fontecave, M.; Pierrel, F. Coq6 Is Responsible for the C4-deamination Reac-tion in Coenzyme Q Biosynthesis in Saccharomyces cerevisiae. J. Biol. Chem. 2015, 290, 24140-24151. [CrossRef]

22. Lapointe, C.P.; Stefely, J.A.; Jochem, A.; Hutchins, P.D.; Wilson, G.M.; Kwiecien, N.W.; Coon, J.J.; Wickens, M.; Pagliarini, D.J. Multi-omics Reveal Specific Targets of the RNA-Binding Protein Puf3p and Its Orchestration of Mitochondrial Biogenesis. Cell Syst. 2018, 6, 125-135. [CrossRef]

23. Veling, M.T.; Reidenbach, A.G.; Freiberger, E.C.; Kwiecien, N.W.; Hutchins, P.D.; Drahnak, M.J.; Jochem, A.; Ulbrich, A.; Rush, M.J.; Russell, J.D.; et al. Multi-omic Mitoprotease Profiling Defines a Role for Oct1p in Coenzyme Q Production. Mol. Cell 2017, 68, 970-977. [CrossRef] [PubMed]

24. Vögtle, F.N.; Prinz, C.; Kellermann, J.; Lottspeich, F.; Pfanner, N.; Meisinger, C. Mitochondrial protein turnover: Role of the pre-cursor intermediate peptidase Oct1 in protein stabilization. Mol. Biol. Cell 2011, 22, 2135-2143. [CrossRef]

25. Acosta Lopez, M.J.; Trevisson, E.; Canton, M.; Vazquez-Fonseca, L.; Morbidoni, V.; Baschiera, E.; Frasson, C.; Pelosi, L.; Ras-calou, B.; Desbats, M.A.; et al. Vanillic Acid Restores Coenzyme Q Biosynthesis and ATP Production in Human Cells Lacking COQ6. Oxid. Med. Cell. Longev. 2019, 2019, 3904905. [CrossRef]

26. Xie, L.X.; Ozeir, M.; Tang, J.Y.; Chen, J.Y.; Jaquinod, S.K.; Fontecave, M.; Clarke, C.F.; Pierrel, F. Overexpression of the Coq8 ki-nase in Saccharomyces cerevisiae coq null mutants allows for accumulation of diagnostic intermediates of the coenzyme Q6 biosyn-thetic pathway. J. Biol. Chem. 2012, 287, 23571-23581. [CrossRef] 
27. Xie, L.X.; Williams, K.J.; He, C.H.; Weng, E.; Khong, S.; Rose, T.E.; Kwon, O.; Bensinger, S.J.; Marbois, B.N.; Clarke, C.F. Resveratrol and para-coumarate serve as ring precursors for coenzyme Q biosynthesis. J. Lipid Res. 2015, 56, 909-919. [CrossRef]

28. Gibson, F. Chemical and Genetic Studies on the Biosynthesis of Ubiquinone by Escherichia coli. Biochem. Soc. Trans. 1973, 1, 317-326. [CrossRef]

29. Poon, W.W.; Do, T.Q.; Marbois, B.N.; Clarke, C.F. Sensitivity to treatment with polyunsaturated fatty acids is a general characteristic of the ubiquinone-deficient yeast coq mutants. Mol. Asp. Med. 1997, 18, 121-127. [CrossRef]

30. Poon, W.W.; Marbois, B.N.; Faull, K.F.; Clarke, C.F. 3-Hexaprenyl-4-hydroxybenzoic acid forms a predominant intermediate pool in ubiquinone biosynthesis in Saccharomyces cerevisiae. Arch. Biochem. Biophys. 1995, 320, 305-314. [CrossRef]

31. Marbois, B.N.; Clarke, C.F. The COQ7 gene encodes a protein in saccharomyces cerevisiae necessary for ubiquinone biosynthe-sis. J. Biol. Chem. 1996, 271, 2995-3004. [CrossRef] [PubMed]

32. Sippel, C.J.; Goewert, R.R.; Slachman, F.N.; Olson, R.E. The regulation of ubiquinone-6 biosynthesis by Saccharomyces cere-visiae. J. Biol. Chem. 1983, 258, 1057-1061. [CrossRef]

33. Goewert, R.R.; Sippel, C.J.; Olson, R.E. Identification of 3,4-dihydroxy-5-hexaprenylbenzoic acid as an intermediate in the bio-synthesis of ubiquinone-6 by Saccharomyces cerevisiae. Biochemistry 1981, 20, 4217-4223. [CrossRef]

34. Bradley, M.C.; Yang, K.; Fernández-Del-Río, L.; Ngo, J.; Ayer, A.; Tsui, H.S.; Novales, N.A.; Stocker, R.; Shirihai, O.S.; Barros, M.H.; et al. COQ11 deletion mitigates respiratory deficiency caused by mutations in the gene encoding the coenzyme Q chaperone protein Coq10. J. Biol. Chem. 2020, 295, 6023-6042. [CrossRef]

35. Allan, C.M.; Awad, A.M.; Johnson, J.S.; Shirasaki, D.I.; Wang, C.; Blaby-Haas, C.E.; Merchant, S.S.; Loo, J.A.; Clarke, C.F. Identification of Coq11, a New Coenzyme Q Biosynthetic Protein in the CoQ-Synthome in Saccharomyces cerevisiae. J. Biol. Chem. 2015, 290, 7517-7534. [CrossRef] [PubMed]

36. Tran, U.C.; Clarke, C.F. Endogenous synthesis of coenzyme Q in eukaryotes. Mitochondrion 2007, 7, S62-S71. [CrossRef] [PubMed]

37. He, C.H.; Xie, L.X.; Allan, C.M.; Tran, U.C.; Clarke, C.F. Coenzyme Q supplementation or over-expression of the yeast Coq8 putative kinase stabilizes multi-subunit Coq polypeptide complexes in yeast coq null mutants. Biochim. Biophys. Acta (BBA)-Mol. Cell Biol. Lipids 2014, 1841, 630-644. [CrossRef] [PubMed]

38. Stefely, J.A.; Licitra, F.; Laredj, L.; Reidenbach, A.G.; Kemmerer, Z.A.; Grangeray, A.; Jaeg-Ehret, T.; Minogue, C.E.; Ulbrich, A.; Hutchins, P.D.; et al. Cerebellar Ataxia and Coenzyme Q Deficiency through Loss of Unorthodox Kinase Activity. Mol. Cell 2016, 63, 608-620. [CrossRef] [PubMed]

39. Lohman, D.C.; Forouhar, F.; Beebe, E.T.; Stefely, M.S.; Minogue, C.E.; Ulbrich, A.; Stefely, J.A.; Sukumar, S.; Luna-Sánchez, M.; Jochem, A.; et al. Mitochondrial COQ9 is a lipid-binding protein that associates with COQ7 to enable coenzyme Q biosyn-thesis. Proc. Natl. Acad. Sci. USA 2014, 111, E4697-E4705. [CrossRef] [PubMed]

40. Floyd, B.J.; Wilkerson, E.M.; Veling, M.T.; Minogue, C.E.; Xia, C.; Beebe, E.T.; Wrobel, R.L.; Cho, H.; Kremer, L.S.; Alston, C.L.; et al. Mitochondrial Protein Interaction Mapping Identifies Regulators of Respiratory Chain Function. Mol. Cell 2016, 63, 621-632. [CrossRef] [PubMed]

41. Ashraf, S.; Gee, H.Y.; Woerner, S.; Xie, L.X.; Vega-Warner, V.; Lovric, S.; Fang, H.; Song, X.; Cattran, D.C.; Avila-Casado, C.; et al. ADCK4 mutations promote steroid-resistant nephrotic syndrome through CoQ10 biosynthesis disruption. J. Clin. Investig. 2013, 123, 5179-5189. [CrossRef] [PubMed]

42. Subramanian, K.; Jochem, A.; Le Vasseur, M.; Lewis, S.; Paulson, B.R.; Reddy, T.R.; Russell, J.D.; Coon, J.J.; Pagliarini, D.J.; Nunnari, J. Coenzyme Q biosynthetic proteins assemble in a substrate-dependent manner into domains at ER-mitochondria contacts. J. Cell Biol. 2019, 218, 1353-1369. [CrossRef] [PubMed]

43. Eisenberg-Bord, M.; Tsui, H.S.; Antunes, D.; Fernandez-Del-Rio, L.; Bradley, M.C.; Dunn, C.D.; Nguyen, T.P.T.; Rapaport, D.; Clarke, C.F.; Schuldiner, M. The Endoplasmic Reticulum-Mitochondria Encounter Structure Complex Coordinates Coenzyme Q Biosynthesis. Contact 2019, 2, 1-14. [CrossRef]

44. Tsui, H.S.; Pham, N.V.B.; Amer, B.R.; Bradley, M.C.; Gosschalk, J.E.; Gallagher-Jones, M.; Ibarra, H.; Clubb, R.T.; Blaby-Haas, C.E.; Clarke, C.F. Human COQ10A and COQ10B are distinct lipid-binding START domain proteins required for coenzyme Q function. J. Lipid Res. 2019, 60, 1293-1310. [CrossRef]

45. Fino, C.; Vestergaard, M.; Ingmer, H.; Pierrel, F.; Gerdes, K.; Harms, A. PasT of Escherichia coli sustains antibiotic tolerance and aerobic respiration as a bacterial homolog of mitochondrial Coq10. MicrobiologyOpen 2020, 9, e1064. [CrossRef] [PubMed]

46. Alcázar-Fabra, M.; Trevisson, E.; Brea-Calvo, G. Clinical syndromes associated with Coenzyme Q10 deficiency. Essays Biochem. 2018, 62, 377-398. [CrossRef]

47. Fernández-Del-Río, L.; Kelly, M.E.; Contreras, J.; Bradley, M.C.; James, A.M.; Murphy, M.P.; Payne, G.S.; Clarke, C.F. Genes and lipids that impact uptake and assimilation of exogenous coenzyme Q in Saccharomyces cerevisiae. Free Radic. Biol. Med. 2020, 154, 105-118. [CrossRef]

48. Gutierrez-Mariscal, F.M.; Yubero-Serrano, E.M.; Villalba, J.M.; Lopez-Miranda, J. Coenzyme Q10: From bench to clinic in aging diseases, a translational review. Crit. Rev. Food Sci. Nutr. 2019, 59, 2240-2257. [CrossRef] [PubMed]

49. Yubero, D.; Montero, R.; Santos-Ocaña, C.; Salviati, L.; Navas, P.; Artuch, R. Molecular diagnosis of coenzyme Q10 deficiency: An update. Expert Rev. Mol. Diagn. 2018, 18, 491-498. [CrossRef] [PubMed]

50. Rudney, H.; Parson, W.W. The conversion of p-hydroxybenzaldehyde to the benzoquinone ring of ubiquinone in Rhodospiril-lum rubrum. J. Biol. Chem. 1963, 238, 3137-3138. [CrossRef] 
51. Parson, W.W.; Rudney, H. The biosynthesis of the benzoquinone ring of ubiquinone from p-hydroxybenzaldehyde and phydroxybenzoic acid in rat kidney, Azotobacter vinelandii, and baker's yeast. Proc. Natl. Acad. Sci. USA 1964, 51, 444-450. [CrossRef]

52. Olson, R.E.; Rudney, H. Biosynthesis of Ubiquinone. Vitam. Horm. 1983, 40, 1-43. [CrossRef] [PubMed]

53. Elliott, W.H.; Waller, G.R. Vitamins and Cofactors. In Biochemical Applications of Mass Spectromery; Waller, G.R., Ed.; WileyInterscience: New York, NY, USA, 1973; pp. 499-536.

54. Olson, R.E. Biosynthesis of Ubiquinones in Animals. Vitam. Horm. 1967, 24, 551-574. [CrossRef]

55. Olson, R.E.; Dialamieh, G.H.; Bentley, R.; Springer, C.M.; Ramsey, V.G. Studies on coenzyme Q. Pattern of labeling in coenzyme Q9 after administration of isotopic acetate and aromatic amino acids to rats. J. Biol. Chem. 1965, 240, 514-523. [CrossRef]

56. Siebert, M.; Severin, K.; Heide, L. Formation of 4-hydroxybenzoate in Escherichia coli: Characterization of the ubiC gene and its encoded enzyme chorismate pyruvate-lyase. Microbiology 1994, 140, 897-904. [CrossRef]

57. Nichols, B.P.; Green, J.M. Cloning and sequencing of Escherichia coli ubiC and purification of chorismate lyase. J. Bacteriol. 1992, 174, 5309-5316. [CrossRef] [PubMed]

58. Payet, L.A.; Leroux, M.; Willison, J.C.; Kihara, A.; Pelosi, L.; Pierrel, F. Mechanistic details of early steps in coenzyme Q biosyn-thesis pathway in yeast. Cell Chem. Biol. 2016, 23, 1241-1250. [CrossRef]

59. Stefely, J.A.; Kwiecien, N.W.; Freiberger, E.C.; Richards, A.L.; Jochem, A.; Rush, M.J.P.; Ulbrich, A.; Robinson, K.P.; Hutchins, P.D.; Veling, M.T.; et al. Mitochondrial protein functions elucidated by multi-omic mass spectrometry profiling. Nat. Biotechnol. 2016, 34, 1191-1197. [CrossRef]

60. Valera, M.J.; Boido, E.; Ramos, J.C.; Manta, E.; Radi, R.; Dellacassa, E.; Carrau, F. The Mandelate Pathway, an Alternative to the Phenylalanine Ammonia Lyase Pathway for the Synthesis of Benzenoids in Ascomycete Yeasts. Appl. Environ. Microbiol. 2020, 86, e00701-20. [CrossRef] [PubMed]

61. Valera, M.J.; Zeida, A.; Boido, E.; Beltran, G.; Torija, M.J.; Mas, A.; Radi, R.; Dellacassa, E.; Carrau, F. Genetic and transcriptomic evidences suggest ARO10 genes are involved in benzenoid biosynthesis by yeast. Yeast 2020, 37, 427-435. [CrossRef] [PubMed]

62. Robinson, K.P.; Jochem, A.; Johnson, S.E.; Reddy, T.R.; Russell, J.D.; Coon, J.J.; Pagliarini, D.J. Defining intermediates and redun-dancies in coenzyme $Q$ precursor biosynthesis. J. Biol. Chem. 2021, 296, 100643. [CrossRef]

63. Luengo, A.; Li, Z.; Gui, D.Y.; Sullivan, L.B.; Zagorulya, M.; Do, B.T.; Ferreira, R.; Naamati, A.; Ali, A.; Lewis, C.A.; et al. Increased demand for $\mathrm{NAD}^{+}$relative to ATP drives aerobic glycolysis. Mol. Cell 2021, 81, 691-707.e6. [CrossRef] [PubMed]

64. Olson, R.E.; Bentley, R.; Aiyar, A.S.; Dialameh, G.H.; Gold, P.H.; Ramsey, V.G.; Springer, C.M. Benzoate derivates as intermedi-ates in the biosynthesis of the coenzyme Q in the rat. J. Biol. Chem. 1963, 238, 3146-3148. [CrossRef]

65. Enríquez, J.A.; Sánchez-Cabo, F.; Vázquez, J. Hypothesis Driven versus Hypothesis-free: Filling the Gaps in CoQ Biosynthesis. Cell Metab. 2016, 24, 525-526. [CrossRef]

66. Mehere, P.; Han, Q.; Lemkul, J.A.; Vavricka, C.J.; Robinson, H.; Bevan, D.R.; Li, J. Tyrosine aminotransferase: Biochemical and structural properties and molecular dynamics simulations. Protein Cell 2010, 1, 1023-1032. [CrossRef] [PubMed]

67. Soubeyrand, E.; Kelly, M.; Keene, S.A.; Bernert, A.C.; Latimer, S.; Johnson, T.S.; Elowsky, C.; Colquhoun, T.A.; Block, A.K.; Basset, G.J. Arabidopsis 4-COUMAROYL-COA LIGASE 8 contributes to the biosynthesis of the benzenoid ring of coenzyme Q in pe-roxisomes. Biochem. J. 2019, 476, 3521-3532. [CrossRef]

68. Block, A.; Widhalm, J.R.; Fatihi, A.; Cahoon, R.E.; Wamboldt, Y.; Elowsky, C.; Mackenzie, S.A.; Cahoon, E.B.; Chapple, C.; Dudareva, N.; et al. The Origin and Biosynthesis of the Benzenoid Moiety of Ubiquinone (Coenzyme Q) in Arabidopsis. Plant Cell 2014, 26, 1938-1948. [CrossRef]

69. Soubeyrand, E.; Johnson, T.S.; Latimer, S.; Block, A.; Kim, J.; Colquhoun, T.A.; Butelli, E.; Martin, C.; Wilson, M.A.; Basset, G.J. The Peroxidative Cleavage of Kaempferol Contributes to the Biosynthesis of the Benzenoid Moiety of Ubiquinone in Plants. Plant Cell 2018, 30, 2910-2921. [CrossRef]

70. Tsao, R. Chemistry and Biochemistry of Dietary Polyphenols. Nutrients 2010, 2, 1231-1246. [CrossRef]

71. Zhang, H.; Tsao, R. Dietary polyphenols, oxidative stress and antioxidant and anti-inflammatory effects. Curr. Opin. Food Sci. 2016, 8, 33-42. [CrossRef]

72. Hatia, S.; Septembre-Malaterre, A.; Le Sage, F.; Badiou-Bénéteau, A.; Baret, P.; Payet, B.; Lefebvre d'Hellencourt, C.; Gonthier, M.P. Evaluation of antioxidant properties of major dietary polyphenols and their protective effect on 3T3-L1 preadipocytes and red blood cells exposed to oxidative stress. Free Radic. Res. 2014, 48, 387-401. [CrossRef] [PubMed]

73. Del Rio, D.; Rodriguez-Mateos, A.; Spencer, J.P.; Tognolini, M.; Borges, G.; Crozier, A. Dietary (Poly) phenolics in Human Health: Structures, Bioavailability, and Evidence of Protective Effects Against Chronic Diseases. Antiox. Redox Signal. 2013, 18, 1818-1892. [CrossRef] [PubMed]

74. Kuršvietienè, L.; Stanevičienè, I.; Mongirdienè, A.; Bernatonienè, J. Multiplicity of effects and health benefits of resveratrol. Medicina 2016, 52, 148-155. [CrossRef]

75. Fernández-del-Río, L.; Nag, A.; Casado, E.G.; Ariza, J.; Awad, A.M.; Joseph, A.I.; Kwon, O.; Verdin, E.; de Cabo, R.; Schneider, C.; et al. Kaempferol increases levels of coenzyme Q in kidney cells and serves as a biosynthetic ring precursor. Free Radic. Biol. Med. 2017, 110, 176-187. [CrossRef] [PubMed]

76. Kozłowska, A.; Szostak-Wegierek, D. Flavonoids—food sources and health benefits. Rocz. Państw. Zakładu Hig. 2014, 65, 79-85. 
77. Soubeyrand, E.; Latimer, S.; Bernert, A.C.; Keene, S.A.; Johnson, T.S.; Shin, D.; Block, A.K.; Colquhoun, T.A.; Schäffner, A.R.; Kim, J.; et al. 3-O-glycosylation of kaempferol restricts the supply of the benzenoid precursor of ubiquinone (Coenzyme Q) in Arabidopsis thaliana. Phytochemistry 2021, 186, 112738. [CrossRef]

78. Fernández-del-Río, L.; Soubeyrand, E.; Basset, G.J.; Clarke, C.F. Metabolism of the Flavonol Kaempferol in Kidney Cells Liber-ates the B-ring to Enter Coenzyme Q Biosynthesis. Molecules 2020, 25, 2955. [CrossRef] [PubMed] 